\title{
The difference between tissue oxygenation markers and development of acute kidney injury in minimally transfused and non-transfused cardiac surgery patients
}

\author{
Büşra Tezcan, Demet Bolukbası, Alev Saylan, Sema Turan, Sultan Sevim Yakın, Dilek Kazancı \\ Turkiye Yuksek Ihtisas Education and Research Hospital - Ankara (Turkey)
}

Goal of Study: To compare AKI development and tissue oxygenation by evaluating ScvO2, lactate, venoarterial $\mathrm{PCO} 2$ difference $(\triangle \mathrm{PCO} 2)$ in minimally transfused and non-transfused cardiac surgery patients.

Materials and Methods: One hundred twentyseven ASA I-II elective coronary artery and valvular heart surgery patients without major intraoperative complications were included in this prospective nonrandomized study. Blood transfusions were driven primarily by hemoglobin levels $(7.0 \mathrm{~g} / \mathrm{dL}$ during cardiopulmonary bypass(CPB) and $8.0 \mathrm{~g} / \mathrm{dL}$ at other times). Patients were divided into two groups: Minimally(1-2 U) Transfused(Group T) and Non-Transfused(Group NT). ScvO2, lactate, $\triangle \mathrm{PCO} 2$ levels were evaluated after anesthesia induction(T1) and at the end of the operation(T2) and compared between the groups. Postoperative Grade I AKI was defined as increase in serum creatinine of more than or equal to $0.3 \mathrm{mg} / \mathrm{dl}$. The lengths of mechanical ventilation and ICU period, development of Grade I AKI and 28 days mortality were recorded and compared between the groups. Chi-square, Fisher's Exact, Student's T, Paired samples T, Mann Whitney-U, Wilcoxon Signed ranks tests were used to compare categoric and scale variables between two groups for statistical analysis.

Results and Discussion: The operation, CPB, cross clamp times, hemodynamic variables, urine output were similar between two groups. Increment rate of lactate and $\triangle \mathrm{PCO} 2$ levels in Group $\mathrm{T}$ were significantly higher than those levels in Group NT. Twentytwo patients (17.5\%) showed AKI and 4 patients $(3.1 \%)$ died in hospital. Group $T$ patients had more $(28,0 \%)$ postoperative AKI without statistically significance. Hospital mortality in Group T was higher than those Group NT with significant difference $(p=0.024)$. Patients with lactate $\geq 2$ $\mathrm{mmol} / \mathrm{L}$ at the end of operation showed more AKI than those patients with normal lactate $(24.2 \%$ vs $9.1 \%$, respectively; $\mathrm{p}=0.030$ ). Lactate, $\triangle \mathrm{PCO} 2, \mathrm{ScvO} 2$ levels showed good correlation for the development of AKI in both groups.

Conclusions: We concluded that intraoperative anemia that is treated with 1-2 units of blood transfusion causes impairment of tissue oxygenation and increases risk of postoperative $A K I$ in cardiac surgery. Impairment of renal tissue oxygenation may be one of the possible mechanisms for the development of postoperative AKI.
Table 1. Tissue oxidation parameters $\left(\Delta \mathrm{CO}_{2}, \mathrm{~S}_{\mathrm{cv}} \mathrm{O}_{2}\right.$ and Lactate $)$ according to groups

\begin{tabular}{|c|c|c|c|}
\hline$\Delta \mathrm{CO}_{2}$ & $\begin{array}{c}\text { T1 } \\
\text { Mean+Std.Dev; (Median; Min-Max) }\end{array}$ & $\begin{array}{c}\text { T2 } \\
\text { Mean+Std.Dev; (Median; Min-Max) }\end{array}$ & $\mathbf{P}$ \\
\hline Group NT & $6.1 \pm 2.8 ; \quad(6.1 ; 0.1-14.5)$ & $6.1 \pm 3.4 ; \quad(6.0 ; 0.4-20.3)$ & $0.929>0.05$ \\
\hline Group T & $4.9 \pm 2.9 ; \quad(5.2 ; 0.8-10.1)$ & $8.8 \pm 7.1 ; \quad(7.4 ; 0.8-31.9)$ & $0.018<0.05$ \\
\hline Total & $5.8+2.8 ; \quad(5.8 ; 0.1-14.5)$ & $6.6 \pm 4.5 ; \quad(6.2 ; 0.4-31.9)$ & $0.264>0.05$ \\
\hline $\mathrm{P}$ & $0.073>0.05$ & $0.057>0.05$ & - \\
\hline \multicolumn{4}{|l|}{$\mathrm{S}_{\mathrm{cV}} \mathrm{O}_{2}$} \\
\hline Group NT & $73.2 \pm 9.0 ; \quad(74.5 ; 45.5-89.8)$ & $64.3 \pm 9.6 ; \quad(63.0 ; 36.8-85.0)$ & $0.000<0.001$ \\
\hline Group T & $71.9 \pm 10.1 ; \quad(71.7 ; 54.0-88.8)$ & $62.3 \pm 9.7 ; \quad(62.0 ; 40.4-79.6)$ & $0.003<0.01$ \\
\hline Total & $72.9 \pm 9.2 ; \quad(73.9 ; 45.5-89.8)$ & $63.9 \pm 9.6 ; \quad(62.7 ; 36.8-85.0)$ & $0.000<0.001$ \\
\hline $\mathbf{P}$ & $0.522>0.05$ & $0.371>0.05$ & - \\
\hline \multicolumn{4}{|l|}{ Lactate } \\
\hline Group NT & $1.1+0.5 \quad(1.0 ; 0.2-2.7)$ & $2.2 \pm 1.1 \quad(2.0 ; 0.7-5.6)$ & $0.000<0.001$ \\
\hline Group T & $1.0 \pm 0.3 \quad(1.0 ; 0.6-1.8)$ & $2.9 \pm 1.6 \quad(2.1 ; 0.9-7.0)$ & $0.000<0.001$ \\
\hline Total & $1.0 \pm 0.4 \quad(1.0 ; 0.2-2.7)$ & $2.3+1.2 \quad(2.0 ; 0.7-7.0)$ & $0.000<0.001$ \\
\hline $\begin{array}{r}\text { P Between } \\
\text { Groups }\end{array}$ & $0.566>0.05$ & $0.009<0.01$ & - \\
\hline
\end{tabular}

Table 2. Comparison of variations in $\Delta \mathrm{CO}_{2}, \mathrm{~S}_{\mathrm{cv}} \mathrm{O}_{2}$, lactate

\begin{tabular}{|c|c|}
\hline$\Delta\left(\Delta \mathrm{CO}_{2}\right)$ & Mean+Std.Dev;(Median; Min-Max) \\
\hline Group NT & $1.7 \pm 9.0 \quad(0.0 ;-0.9-64.0)$ \\
\hline Group T & $(0.4 ;-0.9-19.4)$ \\
\hline Total & $(0.1 ;-0.9-64.0)$ \\
\hline $\mathrm{p}$ & $0.015<0.05$ \\
\hline$\Delta \mathrm{S}_{\mathrm{cv}} \mathrm{O}_{2}$ & Mean+Std.Dev;(Median;Min-Max) \\
\hline Group NT & 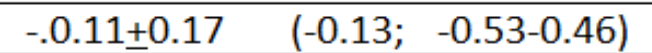 \\
\hline Group T & $(-0.06 ;-0.48-0.27)$ \\
\hline Total & $(-0.12 ;-0.53-0.46)$ \\
\hline$p$ & $0.591>0.05$ \\
\hline$\Delta$ Lactate & Mean+Std.Dev;(Median;Min-Max) \\
\hline Group NT & $1.3 \pm 1.2 \quad(0.9 ;-0.4-6.0)$ \\
\hline Group T & $2.3 \pm 2.4 \quad(1.6 ;-0.3-10.7)$ \\
\hline Total & $1.5 \pm 1.5 \quad(1.0 ;-0.4-10.7)$ \\
\hline & $0.025<0.05$ \\
\hline
\end{tabular}

Table 3. Distribution of AKI and increase of kreatinine levels according to groups

\begin{tabular}{|c|c|c|c|}
\hline \multicolumn{4}{|c|}{ Distribution of patients according to transfusion and AKI subgroups } \\
\hline Groups & $\mathrm{AKI}+$ & AKI - & TOTAL \\
\hline Group NT & $15(14.9 \%)$ & $86(85.1 \%)$ & $101(80.2 \%)$ \\
\hline Group T & $7(28.0 \%)$ & $18(72.0 \%)$ & $25(19.8 \%)$ \\
\hline Total & $22(17.5 \%)$ & $104(82.5 \%)$ & $126(100.0 \%)$ \\
\hline $\mathrm{p}$ & \multicolumn{3}{|c|}{$0.143>0.05$} \\
\hline \multicolumn{4}{|c|}{ Increase in kreatinine levels according to groups $(\mathrm{mg} / \mathrm{dL})$} \\
\hline $\begin{array}{l}\text { Group NT Mean + SD } \\
\text { (Median; Min-Max) }\end{array}$ & \multicolumn{2}{|c|}{$0.06+0.24(0.05 ;-0.57-0.85)$} & \\
\hline $\begin{array}{l}\text { Group T Mean + SD } \\
\text { (Median; Min-Max) }\end{array}$ & \multicolumn{2}{|c|}{$0.26+0.55(0.09 ;-0.37-1.81)$} & $p: 0.204>0.05$ \\
\hline $\begin{array}{l}\text { Total } \quad \text { Mean + SD } \\
\text { (Median; Min-Max) }\end{array}$ & \multicolumn{2}{|c|}{$0.10+0.10(0.06 ;-0.57-1.81)$} & \\
\hline
\end{tabular}

Table 4. Distribution of one-month mortality of T and NT groups in different AKI subgroups

\begin{tabular}{|l|l|r|r|r|c|}
\hline \multirow{4}{*}{ AKI - } & & \multicolumn{2}{|c|}{ ONE-MONTH MORTALITY } & \multirow{2}{*}{ p } \\
\cline { 2 - 5 } & GROUP & \multicolumn{1}{|c|}{ NO } & \multicolumn{1}{c|}{ YES } & \multicolumn{1}{|c|}{ TOTAL } & \\
\cline { 2 - 5 } & NT & $85(98.8 \%)$ & $1(1.2 \%)$ & $86(82.7 \%)$ & \multirow{2}{*}{$0.318>0.05$} \\
\cline { 2 - 5 } & T & $17(94.4 \%)$ & $1(5.6 \%)$ & $18(17.3 \%)$ & \\
\cline { 2 - 5 } & TOTAL & $102(98.1 \%)$ & $2(1.9 \%)$ & $104(100.0 \%)$ & \\
\hline \multirow{3}{*}{ AKI + } & NT & $15(100.0 \%)$ & $0(0.0 \%)$ & $15(68.2 \%)$ & \multirow{2}{*}{$0.091>0.05$} \\
\cline { 2 - 5 } & T & $5(71.4 \%)$ & $2(28.6 \%)$ & $7(31.8 \%)$ & \\
\cline { 2 - 5 } & TOTAL & $20(90.9 \%)$ & $2(9.1 \%)$ & $22(100.0 \%)$ & \\
\hline \multirow{3}{*}{ TOTAL } & NT & $100(99.0 \%)$ & $1(1.0 \%)$ & $101(80.2 \%)$ & \multirow{2}{*}{$0.024<0.05$} \\
\cline { 2 - 5 } & T & $22(88.0 \%)$ & $3(12.0 \%)$ & $25(19.8 \%)$ & \\
\cline { 2 - 5 } & TOTAL & $122(96.8 \%)$ & $4(3.2 \%)$ & $126(100.0 \%)$ & \\
\hline
\end{tabular}

